\section{The NIH improves its record on women in trials}

Studies released last month show that although the National Institutes of Health (NIH) has been slow to enforce guidelines from its $1993 \mathrm{NIH}$ Revitalization Act, regarding the enrollment of women in clinical trials and the analysis of trial data by gender, it has made progress in this area.

In a paper to be published this month in the Journal of Women's Health and Gender-based Medicine, the authors surveyed scientific literature from four major medical journals-New England Journal of Medicine, the Journal of the American Medical Association, the Journal of the National Cancer Institute, and Circulation - for the years 1993, 1995, 1997 and 1998. They found that approximately one-fifth of the NIH-funded studies published each year failed to include women as research subjects, and only one-third of the studies that did include women analyzed data by sex of the subjects.

However, because clinical trials can take several years from enrollment to completion, this research includes data from studies that would have been initiated before the revitalization act. In fact, an audit of the NIH's clinical research studies during FY97 by the General Accounting Office (GAO) does show improvement-more than half of trial participants were women in that year. Specifically, data for all extramural research protocols funded, which were tracked by the NIH, show that $61.9 \%$ of subjects were women and looking at Phase III studies reveals that only $74.8 \%$ were woman. The report concludes, "In the past decade, $\mathrm{NIH}$ has made significant progress in implementing a strengthened policy on including women in clinical research." These 1997 figures should begin to show up in studies published in the literature this year.

But the news is not all good. The GAO was less flattering about the NIH's implementation of the requirement that trials be designed to permit valid analysis by gender, which can reveal whether interventions affect the sexes differently. It found that the NIH had not adequately enforced this policy and recommends that the director of the NIH ensure that "phase III clinical trials be designed and carried out to allow for the valid analysis of differences between women and men as fully as it implements other elements of the inclusion policy."

This is the second time that the GAO has examined the NIH's inclusion of women in trials. In 1990 it found that the agency had not acted on guidelines it had set in 1986 urging the inclusion of women of child-bearing age in federally funded trials. This led to the 1990 establishment of the NIH Office of Research on Women's Health (ORWH), whose responsibility it is to ensure that women and minorities are included in studies. The ORWH's budget has increased from \$9.4 million in 1993 to around \$20 million in FY00, and this office is now preparing a report on its 1997-1998 activities for the director of the NIH.

The new GAO report was commissioned by senators Tom Harkin (D-IA), Olympia Snowe (R-ME) and Barbara Mikulski (D$\mathrm{MD})$, who are now asking Senator Jim Jeffords (R-VT), chairman of the Committee on Health, Education, Labor and Pensions, to debate the subject in congress. They have also requested that by the year's end the GAO carries out a similar assessment, through the Food and Drug Administration, of the number of women in trials sponsored by the pharmaceutical industry.

\section{Karen Birmingham, London}

\title{
New economic analysis draws big money to malaria
}

At the first sub-Saharan African regional summit meeting on malaria on 25 April, leaders from several African nations called for, and received, vast increases in funding-up to $\$ 1$ billion a year-from developed countries to help fight the disease. "By the end of the two-day summit," says World Health Organization (WHO) spokesman Jim Palmer, "up to $\$ 750$ million in extra funds were made available by Canada, the UK, the US, the World Bank and other sources."

That African leaders were able to ask for and receive such a commitment emphasizes the persuasiveness of a report prepared for the summit by the Harvard University Center for International Development and the London School of Hygiene and Tropical Medicine. Jeffrey Sachs, the Harvard health economist who supervised the preparation of the report, has been an outspoken advocate of restructuring international financial institutions to focus more resources on health-related issues and research (Nature Med. 6, 491; 2000), a theme echoed in the new report, which analyzed the total economic effect of malaria on sub-Saharan Africa.

In the report, Sachs and his colleagues conclude that "the annual loss of [economic] growth from malaria is estimated to range as high as 1.3 percentage points per year. If this loss is compounded for fifteen years, the GNP level in the fifteenth year is reduced by nearly a fifth, and the toll continues to mount with time."

Previous estimates of the economic cost of malaria consisted of simpler static projections based only on lost productivity and direct medical expenses. In the new analysis, the researchers compounded the "malaria growth penalty" over time, and also considered a broader range of factors, including the social cost of pain and suffering and losses to tourism, foreign investment and commerce. Based on the more comprehensive estimates, the economists project that the benefits of controlling the disease are "in the dozens of billions of dollars per year after a few years of malaria control," making a $\$ 1$ billion investment highly cost-effective.

The new money, $\$ 500$ million of which is being provided by the World Bank, will support the ongoing WHO Roll Back Malaria campaign, which seeks to cut the incidence of malaria in Africa in half by 2010. Though WHO sources would not specify exactly how the program's new budget windfall will be divided, the bulk of the funding is expected to go to disease control efforts, such as distributing insecticide-treated bed nets and anti-malarial drugs. A smaller but substantial portion will fund basic research and training in a variety of fields, including epidemiology, ecology, entomology and immunology.

Sachs's report identifies research as a key component of an overall malaria control effort. In addition to studies focused on developing new treatments and vaccines, the report cites a "dire lack of extensive and comparable data about malaria," and calls for more research on trends in incidence and prevalence, epidemic outbreaks, clinical epidemiology and interactions with other diseases.

Alan Dove, Philadelphia 